\title{
Learn-by-Doing and Carbon Dioxide Abatement
}

\author{
Alan S. Manne and Leonardo Barreto
}

RR-04-013

November 2004 



\title{
Learn-by-Doing and Carbon Dioxide Abatement
}

\author{
Alan S. Manne \\ Stanford University, Stanford, CA, USA \\ Leonarod Barreto \\ International Institute for Applied Systems Analysis, Laxenburg, Austria
}

RR-04-013

November 2004

Reprinted from Energy Economics, 26(4):621-633 (2004).

International Institute for Applied Systems Analysis - Schlossplatz 1 - A-2361 Laxenburg • Austria Tel: (+43 2236) 807 • Fax: (+43 2236) 71313•E-mail: publications@iiasa.ac.at•Web: www.iiasa.ac.at 
Research Reports, which record research conducted at IIASA, are independently reviewed before publication. Views or opinions expressed herein do not necessarily represent those of the Institute, its National Member Organizations, or other organizations supporting the work.

Reprinted from Energy Economics, 26(4), Alan S. Manne and Leonardo Barreto, Learn-by-doing and carbon dioxide abatement, pp. 621-633 (2004), with permission from Elsevier. Copyright $\odot 2004$ Elsevier B.V.

All rights reserved. No part of this publication may be reproduced or transmitted in any form or by any means, electronic or mechanical, including photocopy, recording, or any information storage or retrieval system, without permission in writing from the copyright holder. 


\title{
Learn-by-doing and carbon dioxide abatement
}

\author{
Alan S. Manne ${ }^{\mathrm{a}, *}$, Leonardo Barreto ${ }^{\mathrm{b}}$ \\ a Management Science and Engineering, Terman Engineering Center, Stanford University, Stanford, \\ CA 94305-4026, USA \\ ${ }^{\mathrm{b}}$ International Institute for Applied Systems Analysis, Laxenburg, A-2361, Austria
}

Available online 17 June 2004

\begin{abstract}
There are inherent difficulties in solving earn-by-doing (LBD) models. Basic to such models is the idea that the accumulation of experience leads to a lowering of costs.

This paper is intended to explore some of the algorithmic issues in LBD modeling for carbon dioxide abatement. When using a standard algorithm for nonlinear programming, there is no guarantee that a local LBD optimum will also be a global optimum. Fortunately, despite the absence of guarantees, there is a good chance that one of the standard algorithms will produce a global optimum for models of this type - particularly if there is an artful selection of the starting point or of the terminal conditions. Moreover, there is a new procedure named BARON. In the case of small models, a global optimum can be recognized and guaranteed through BARON.

Eventually, it should be possible for BARON or a similar approach to be extended to large-scale LBD models for climate change. Meanwhile, in order to check for local optima, the most practical course may be to employ several different starting points and terminal conditions.

(C) 2004 Elsevier B.V. All rights reserved.
\end{abstract}

Keywords: Learn-by-doing model; Carbon dioxide abatement; BARON

\section{Introduction}

There are inherent difficulties in solving learn-by-doing (LBD) models. Basic to such models is the idea that the accumulation of experience leads to a lowering of costs. This idea goes back to the model of Arrow (1962) - and even earlier to empirical estimates of airframe production costs. Within the context of global climate change, it has been applied by Goulder and Mathai (2000), Gritsevskyi and Nakicenovic (2000), Kydes (1999),

\footnotetext{
* Corresponding author.

E-mail address: ASManne@pacbell.net (A.S. Manne).
} 
Kypreos et al. (2000), Kypreos (2000), Mattsson and Wene (1997), Messner (1997), Seebregts et al. (2000), TEEM (1999) and Van der Zwaan et al. (2002).

This paper is intended to explore some of the algorithmic issues in LBD modeling for carbon dioxide abatement. When using a standard algorithm for convex nonlinear programming, there is no guarantee that a local LBD optimum will also be a global optimum. Fortunately, despite the absence of guarantees, there is a good chance that one of the standard procedures will produce a global optimum for models of this typeparticularly if there is an artful selection of the starting point or of the terminal conditions. Moreover, there is a new algorithm named BARON. In the case of small-size LBD models, a global optimum can be recognized and guaranteed through BARON.

For a general idea of what is involved, see Fig. 1. There are just two decision variables, $x_{1}$ and $x_{2}$. The feasible set consists of a convex polygon: all points within the shaded area $\mathrm{ABCD}$. If the minimand is strictly concave, it can happen that point $\mathrm{A}$ is a local optimum. That is, it has lower costs than the adjacent extreme point $\mathrm{B}$, but it has higher costs than the distant extreme point $\mathrm{C}$. This is illustrated by the two dashed iso-cost lines. The curved line going through $\mathrm{A}$ indicates higher system costs than the curved line going through $\mathrm{C}$. In any case, the minimum system cost lies at one of the extreme points (the vertices) - not between them. (For a rigorous treatment of this proposition, see Hirsch and Hoffman (1961).) Moreover, there may be only a small difference in costs between the extreme points.

In connection with the debate over global climate change, a small example of LBD will be examined in this paper. We will show that occasionally one of the standard algorithms fails, but that BARON is successful in producing a global optimum. Simultaneously with this effort, we are applying some of these ideas to a larger, more realistic model known as

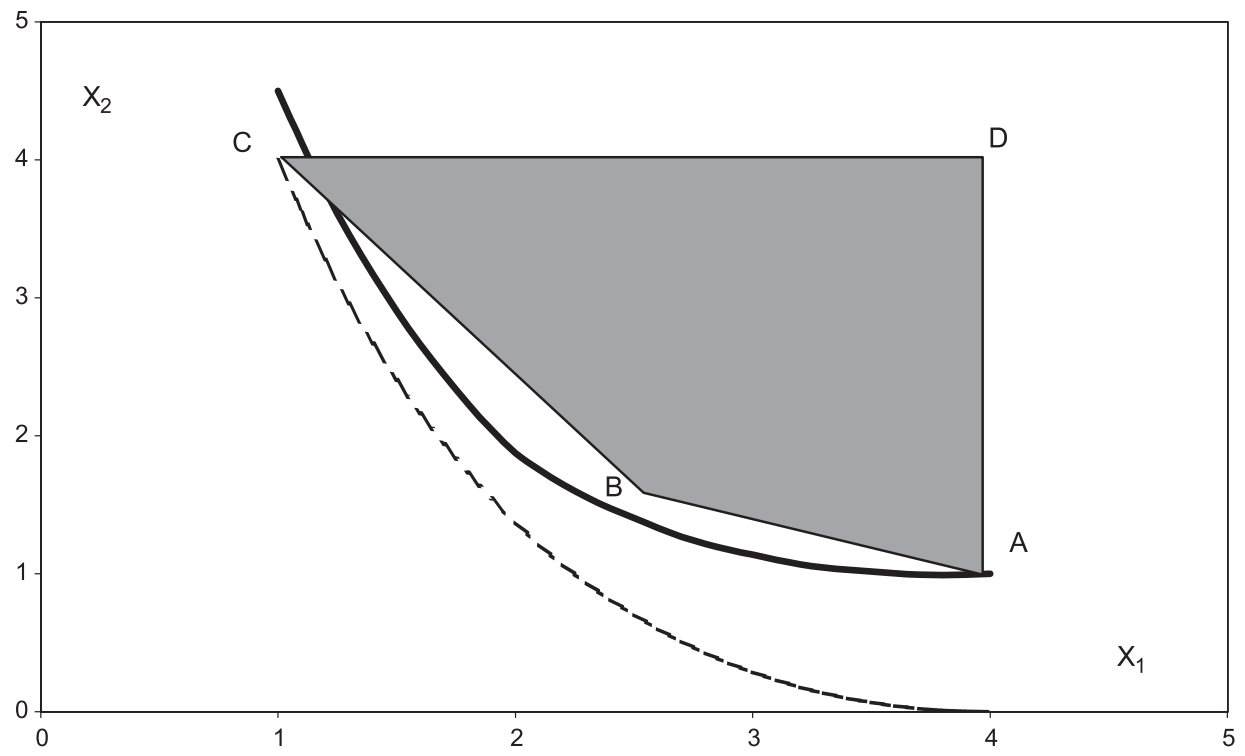

Fig. 1. Two-dimensional example of a local optimum. 
MERGE. Here the standard algorithms produce plausible solutions, but we have to take any steps that we can to ensure that these represent a global rather than a local optimum.

\section{The BARON algorithm}

For details on BARON, see Sahinidis (2000) and http://archimedes.scs.uiuc.edu. According to: http://www.gamsworld:

BARON is a computational system for solving non convex optimization problems to global optimality. Purely continuous, purely integer, and mixed-integer nonlinear problems can be solved with the software. The Branch And Reduce Optimization Navigator derives its name from its combining interval analysis and duality in its reduce arsenal with enhanced branch and bound concepts as it winds its way through the hills and valleys of complex optimization problems in search of global solutions.

BARON is a tool that allows for the identification of globally optimal solutions. It combines range reduction techniques with an enhanced branch and bound algorithm. This combination gives the name to the algorithm: Branch and Reduce. The Branch and Bound algorithm is applied to a (generally convex) relaxation of the original non-convex problem. In each node, a relaxed version of the original problem is solved. If this is a minimization, its solution provides a lower bound for the original non-convex problem. Using this solution as the starting point (or, if available, a better starting point can be used), the original problem is solved and an upper bound for the global optimal solution is found. If the gap between the upper and lower bounds is not small enough, the feasible region is divided in parts. A new relaxed problem is solved for each subdivision and new lower and upper bounds for the global optimum are computed.

The range reduction techniques help to restrict the search space and reduce the relaxation gap. They are applied to every sub-problem of the branch-and-bound search tree in pre- and post-processing steps, helping to improve the performance of the bounding procedure at every node of the tree. Different types of reduction tests can be applied according to the form of the problem. Optimality- and feasibility-based range reduction tests are possible. Optimality-based range reduction uses the optimal (dual) solution of the relaxed problem to reduce the range of constraints and variables. Feasibility-based range reduction uses heuristic procedures to generate constraints that eliminate infeasible portions of the solution. These constraints approximate the solution of optimization problems that generate improved bounds for the problem variables.

\section{The LBD perspective}

For an example of LBD, see Fig. 2, copied from International Energy Agency (2000). This reports average unit costs (1990 ECU per kilowatt-hour) for a series of alternative electricity producing technologies in the European Union, 1980-1995. The vertical axis refers to unit costs; the horizontal axis refers to cumulative electricity production at 


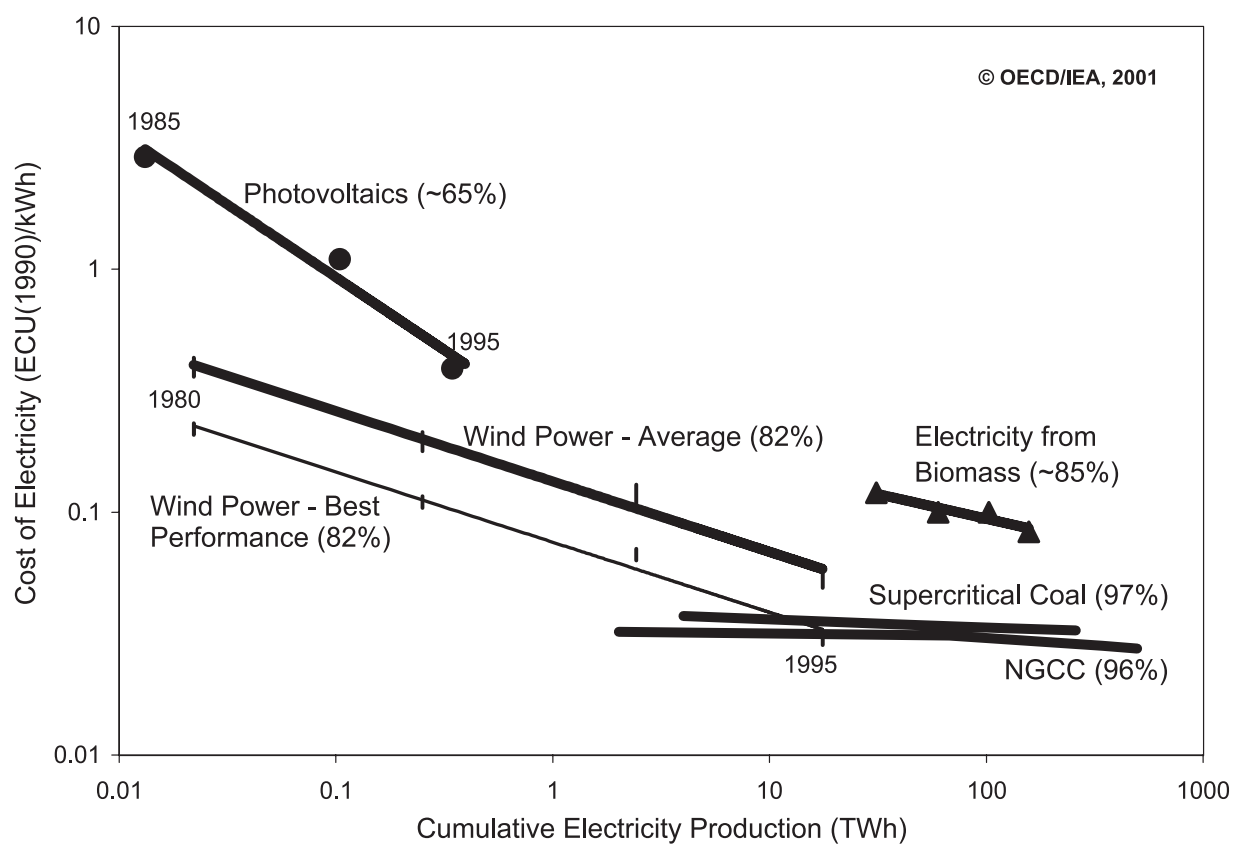

Fig. 2. Electric technologies in EU 1980-1995.

successive dates. With greater "experience" (cumulative production), there is a pronounced tendency for a decline in the unit costs of novel technologies such as photovoltaics and wind power, but there is no obvious decline in the unit costs of more conventional methods such as supercritical coal and NGCC (natural gas-combined cycle). Significantly enough, nuclear power is not plotted on this diagram. If it were plotted, it would almost surely illustrate an increase in unit costs with additional experience - and with additional concerns about reactor safety.

In Fig. 2, note that the newer technologies tend to be higher in unit costs than the conventional ones. If investors based all their decisions on immediate costs, there would be little tendency to support the newer technologies that are currently more expensive. Their cumulative experience is too small, and they could be "locked out" permanently. This is the rationale for public intervention in the market. LBD entails the acceptance of high near-term costs in return for an expected lowering of future costs. It is an investment choice, and it depends critically upon the rate of discount.

Associated with each technology, Fig. 2 shows a "progress ratio" entered in parentheses. This measures the percentage decline in unit costs that is associated with a doubling of experience. In the case of wind, for example, this parameter is shown as $82 \%$. That is, $2^{\operatorname{lrn}}=82 \%$. Therefore, the exponent $\operatorname{lrn}=-0.29$. This exponent is one of the essential parameters that is entered into an LBD model.

So far, so good. The next analytical issue is the measurement of cumulative experience. Should this be limited to the European Union (as in Fig. 2)? Or should it also take account of efforts elsewhere - in Japan, the USA, etc.? This is not an easy question to answer. In a 
global economy, technological experience diffuses widely. It is quite possible that there is a more rapid flow of information between the European and US branches of a given company than between the European branches of different companies.

The geographical range of diffusion is one issue. Another is the measurement of cumulative experience associated with the leftmost point along each curve. If, for example, there is no experience with wind power reported before 1980, how do we measure the cumulative experience at this initial date? The initial cumulative experience is an estimate that must be made thoughtfully, and there are no easy answers. Even in the year 2000, the production of wind and solar electricity provides just a small percentage of the total.

\section{A small-scale model of electricity choices}

In order to develop a small-scale model of electricity choices, consider the options that are available to the world as a whole. It will be supposed that the world plans to meet the total electricity demands implied by the "reference case" of MERGE. This is a multiregion, multi-technology model for estimating the costs of regional and global greenhouse gas reductions. It is based upon a bottom-up view of energy supplies and a top-down view of energy demands. For details on MERGE, see the website: http:/www.stanford.edu/ group/MERGE.

To meet the reference case demands, suppose that there are just three technologies available:

1. defender: the average type of unit on line in the year 2000; a predominantly fossil mix of technologies, but also includes hydroelectric and nuclear; it is not subject to LBD.

2. challenger: the initial challenger-the average type of carbon-free technology available in 2000; this is high cost and subject to learning along the lines of the LBD model; and

3. advanced: an advanced challenger - the average type of carbon-free technology that might become available in 2050; this is lower-cost and also subject to the endogenous type of learning.

Let the decision variables $X_{j, t}$ denote the quantity of electric energy (trillion kilowatthours) produced by technology $j$ in period $t$ (where the time periods refer to successive decades during the $21 \mathrm{st}$ century). Together, the three technologies must meet the projected electricity demands. If one is not concerned about carbon accumulation, one could meet these demands solely through technology 1 (the low-cost, predominantly fossil-based option). If one is concerned about reducing carbon, there will be a role for the higher-cost carbon-free technologies. The earlier one has the advantage of being available immediately, but the later one has the advantage of being potentially lower in costs. It might, for example, represent nuclear or fusion. Or it might represent advanced developments of wind or of photovoltaic solar-or fossil fuel plants with carbon capture and sequestration. 
To express the condition that total demands must be met by a combination of these three technologies, there is first the supply-demand balance constraint:

$$
X_{1, t}+X_{2, t}+X_{3, t} \geq E_{t},
$$

where $E_{t}$ denotes the demands in decade $t$.

Next, there are the constraints that none of these technologies may expand too rapidly. To illustrate this idea concretely, suppose that a new technology cannot supply more than $1 \%$ of the market during the first decade in which it is introduced, and that it cannot expand much more rapidly than a factor of four during subsequent decades. We then have:

$$
X_{j, t+1} \leq .01 E_{t}+4 X_{j, t} \quad(\text { for all } j, t)
$$

Similarly, to ensure that technologies are not replaced too rapidly, we impose a maximum annual decline rate of $3 \%$ per year. For intervals of a decade, this works out as follows:

$$
X_{j, t+1} \geq(1 / 1.03)^{10} X_{j, t} \quad(\text { for all } j, t)
$$

In order to keep track of cumulative carbon emissions from the electric power sector, we take the average of emissions at the beginning and the end of each decade. Cumulative emissions through decade $t$ are represented by the decision variable. $\mathrm{CARB}_{t}$. They are proportional to the output of technology 1 (the predominantly fossil fuel defender):

$$
\mathrm{CARB}_{t+1}=\mathrm{CARB}_{t}+5 \operatorname{cec}\left(X_{1, t}+X_{1, t+1}\right)
$$

where cec represents the average carbon emission coefficient during the year 2000. Under a "business-as-usual" scenario, the cumulative emissions would be roughly 700 billion tons during the 21 st century. To illustrate a low-carbon scenario-but one in which there is no immediate need for abatement-we impose an upper bound of 400 billion tons on the terminal year cumulative emissions, $\mathrm{CARB}_{\mathrm{T}}$.

It is assumed that learning costs depend upon the cumulative production experience for each of the technologies. Let the decision variables $Y_{j, t}$ represent this experience. Basing these variables upon an average of the production at the beginning and end of each decade, we have:

$$
Y_{j, t+1}=Y_{j, t}+5\left(X_{j, t}+X_{j, t+1}\right) \quad(\text { for all } j, t)
$$

The objective function is expressed as one of minimizing the present value of costssubject to meeting the supply-demand constraints (1), the expansion and decline constraints (2) and (3), the cumulative carbon constraints (4), the cumulative production experience (5), and both upper and lower bounds on individual variables. In order to employ a market-oriented criterion, we let $\mathrm{pv}_{t}$ (the present value factor for period $t$ ) be based upon a $5 \%$ real rate of return on capital. This is intended to be net of inflation, and represents a before-tax rate of return. Let the decision variable PVC denote the present value of costs throughout the 21 st century. For each time period and each technology, we 
Table 1

Illustrative values of the cost parameters

\begin{tabular}{|c|c|c|c|}
\hline \multirow[t]{2}{*}{ Technology $j$} & 1 & 2 & 3 \\
\hline & Defender & Challenger & Advanced \\
\hline Static cost coefficients, cost $j, \$$ per thousand $\mathrm{kWh}$ & 40 & 30 & 30 \\
\hline Initial learning cost coefficients, inlc,$\$$ per thousand $\mathrm{kWh}$ & 0 & 50 & 10 \\
\hline Initial accumulated experience, $\operatorname{acc}_{j}$, trillion $\mathrm{kWh}$ & 1 & 1 & 1 \\
\hline Learning exponent, $\operatorname{lrn}_{j}$ & n.a. & -0.2 & -0.2 \\
\hline
\end{tabular}

then have two cost components. The first may be termed "static" and the second "dynamic".

$$
\mathrm{PVC}=\sum_{t} \mathrm{pv}_{t}\left\{\sum_{t} \operatorname{cost}_{j} X_{j, t}+\sum_{j} \operatorname{incl}_{j} X_{j, t}\left[\frac{Y_{j, t}}{\operatorname{acc}_{j}}\right]^{\ln _{j}}\right\}
$$

That is, the static terms are proportional to the cost ${ }_{j}$ factors. These provide a lower bound on the average cost of each technology. The dynamic terms depend upon the cumulative learning experience. This in turn depends upon the $Y_{j, t}$ decision variables - and also upon three parameters: the initial learning cost coefficient inlc ${ }_{j}$, the initial experience $\mathrm{acc}_{j}$, and the learning exponent $\operatorname{lrn}_{j}$. Table 1 shows illustrative values of these individual parameters. In this case, the costs of the fossil fuel defender remain constant over time - at $\$ 40$ per thousand $\mathrm{kWh}$. The learning exponent is "n.a." (not applicable) in this case. Initially, the first challenger's costs are twice the level of the defender: $30+50=\$ 80$ per thousand $\mathrm{kWh}$. These costs decline over time with cumulative experience. The advanced challenger has lower initial costs, but does not become available until 2050.

Note that the initial accumulated experience parameters $\operatorname{acc}_{j}$ must be chosen with care. Over time - with cumulative experience - the costs of all three technologies will decline toward the limits imposed by the static cost factors. The parameters acc ${ }_{j}$ must be checked for their comparability with the values of the cumulative production variables $Y_{j, t}$ during the initial decades of the 21 st century.

The reader could experiment with other parameters. From earlier work, for example, we know that the optimal solution is highly sensitive to the learning exponent, $\operatorname{lrn}_{j}$.

Just as in the two-dimensional example (Fig. 1), the constraint set of this problem is a convex polyhedron. The minimand is concave. A solution must therefore lie at one or another of the extreme points of the polyhedral constraint set. However, it is not sufficient to check adjacent extreme points. One must somehow be able to verify that distant extreme points are also handled. This is the role played by BARON.

\section{Numerical results from the small-scale model}

Fig. 3 shows the percentages supplied by each of the three technologies in the global, minimum-cost solution to this problem. Each technology follows a unimodal path. That is, there is at most one maximum point for its deployment. There are distinct phases in which one or another expansion/decline constraint is active. The first challenger is not introduced 


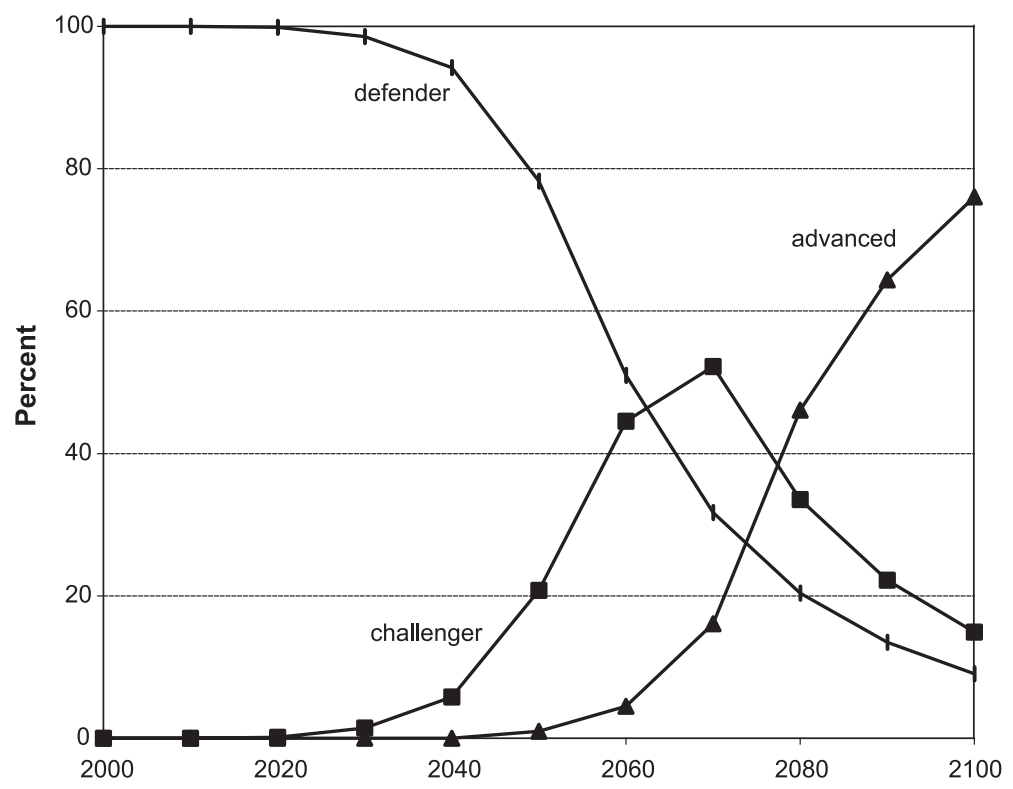

Fig. 3. Percentages of demand supplied by alternative technologies.

immediately in 2010. With a cumulative carbon constraint of 400 billion tons, there is enough slack in the system so that the challenger does not need to enter until 2020. Thereafter, it expands at a maximum rate until 2050. The fossil defender begins to decline after 2040. In 2050, the advanced challenger begins to enter at its maximum rate, and after 2070, the earlier challenger begins its decline.

Along with these introduction patterns, there is a distinct pattern of learning costs. To see how the average unit learning costs change with cumulative experience, see Fig. 4. There, results are reported for two alternative values of the accumulated initial learning experience parameter. When $\operatorname{acc}_{j}=1.0$, we obtain the upper experience curve. In this case, it is optimal to wait until 2020 before introducing the challenger. Alternatively, if $\operatorname{acc}_{j}=0.1$, this provides a more attractive initial point for the challenger. The same unit costs are attained with less experience. In turn, this creates an incentive for more rapid deployment of the challenger - and an earlier date at which costs begin to be lowered.

Each of the experience curves is determined by the three dynamic LBD parameters listed in Table 1. The vertical distance of the 2000 value is the initial learning cost coefficient, inlc ${ }_{j}$. The horizontal distance of the 2000 value is the initial accumulated experience, $\mathrm{acc}_{j}$. And the slope of the experience curves (on a log-log scale) is given by the learning exponent, $\operatorname{lrn}_{j}$. The rate at which we progress down the experience curve is determined by the endogenous learning process. The less expensive the challenger, the more rapidly it is deployed.

To put things into perspective, it is useful to examine Fig. 5. This shows the total of the static plus the dynamic learning costs for the challenger. Both cost curves begin at the same point in 2000 - at twice the level of the defender technology-but they diverge thereafter. Throughout the 21 st century, there is no date at which the upper curve lies 


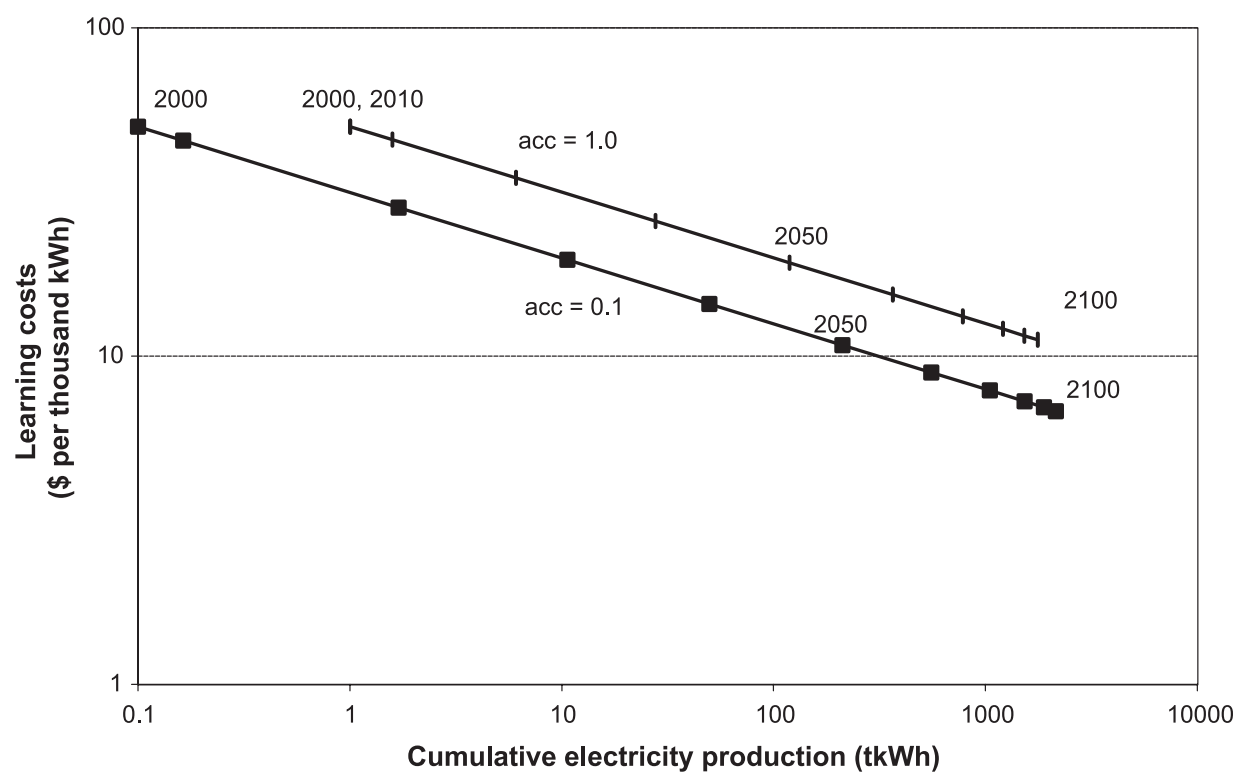

Fig. 4. Experience curves for challenger-alternative values of initial experience, acc.

below the costs of the defender ( $\$ 40$ per thousand $\mathrm{kWh}$ ). Without a carbon constraint, there would be no rationale to introduce the challenger. With the lower curve, however, the challenger's costs lie below those of the defender from 2050 onward. Under these

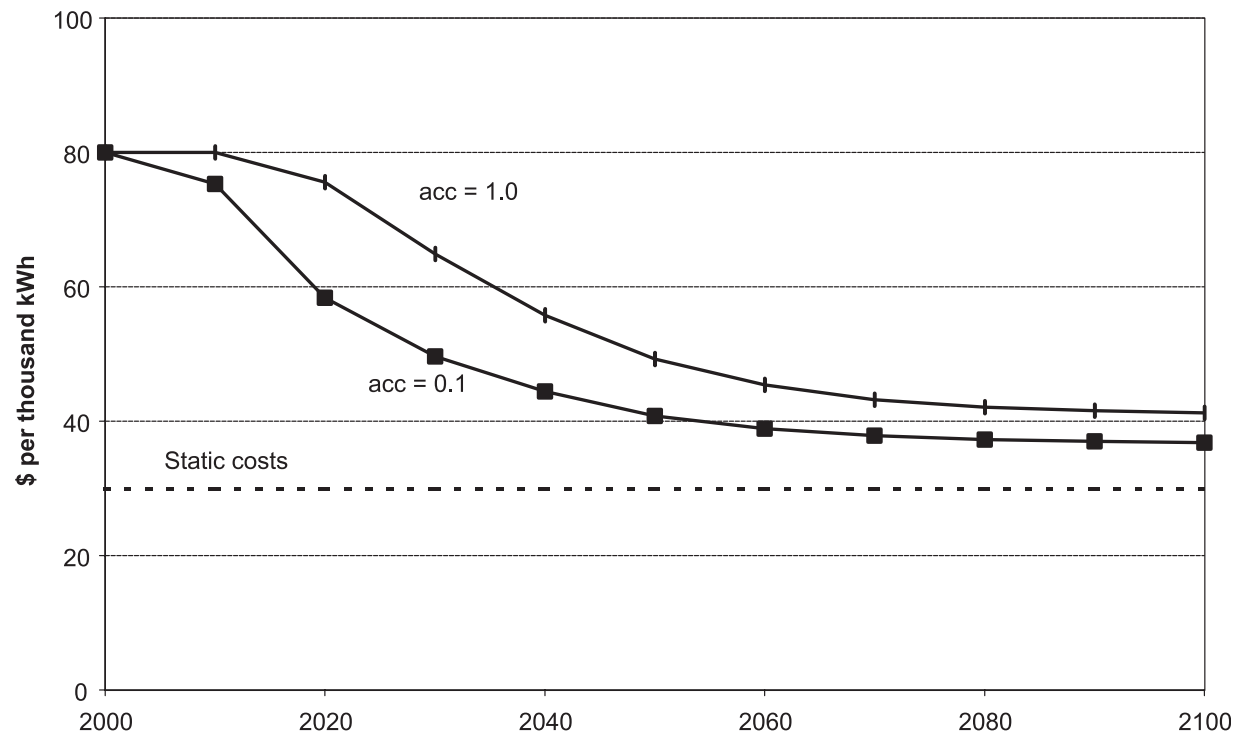

Fig. 5. Static + learning costs for challenger. 
circumstances, the challenger is introduced at the maximum rate from the earliest date that it becomes available, and the 400 billion ton carbon constraint becomes inactive.

These projections should not be taken literally, but they do indicate that this type of LBD model has a tendency toward "bang-bang" behavior. That is, a technology may not be introduced immediately when it becomes available. When it is introduced, however, it tends to enter at a maximum growth rate, and eventually to be phased out at a maximum decline rate.

When $\operatorname{acc}_{j}=1.0$, the results of BARON are duplicated by two standard convex nonlinear programming algorithms: CONOPT3 and MINOS5. There is a coincidence between the local and the global optimum. How often does this occur? Not always. For example, when we take the same model but eliminate the carbon constraint, we obtain two different solutions. CONOPT3 generates the same global optimum as BARON, but MINOS5 generates a very different local optimum.

Without a carbon constraint, the global optimum is one in which the defender supplies all of the demands through 2040. From 2050 onward, the advanced low-cost challenger then expands at the maximum rate. The locally optimal solution is one in which the defender supplies the world's demands throughout the entire horizon. The other two technologies are both locked out. With a different starting point, MINOS5 produces still a different solution, but again one that is not a global optimum. In all the sensitivity analyses that we have conducted, CONOPT3 has duplicated the same globally optimal solution as BARON, but MINOS5 has produced a number of local optima.

Caveat: These experiments are not conclusive. To our knowledge, there is no theoretical reason for the superiority of one or another of these standard methods when the minimand is concave. Eventually, it should be possible for BARON or a similar approach to be extended to large-scale LBD models. Meanwhile, in order to check for local optima, the most practical course is to resort to heuristics. One possibility would be to apply several different nonlinear programming algorithms - and several different starting solutions with each of them.

\section{An alternative approach-terminal conditions}

Another possibility is to experiment with alternative terminal conditions. This is the approach that has been applied at a large scale in connection with the MERGE model. To see how this works, consider Fig. 6. This is based on the small-scale numerical model described in this paper, but the inlc parameter for the advanced technology has been increased to $\$ 20 / \mathrm{MWh}$ to provide a clearer example.

For the initial challenger, the cumulative experience through 2100 is shown on the vertical axis, $Y(\mathrm{chl}, 2100)$. The cumulative experience for the advanced challenger is shown on the horizontal axis, $Y(\mathrm{adv}, 2100)$. Both of these variables are expressed in trillion kilowatt-hours. The feasible combinations of these two variables are shown within the shaded polygon. The lower edge of this area is the $45^{\circ}$ line determined by the cumulative carbon emissions constraint. The upper edge is implied by equalities in the supplydemand balances, constraints (1). Again this is a $45^{\circ}$ line. The leftmost edge is governed by the lower bound constraint on $Y(\mathrm{adv}, 2100)$, and the rightmost edge by the combined effect of the expansion and decline constraints. 


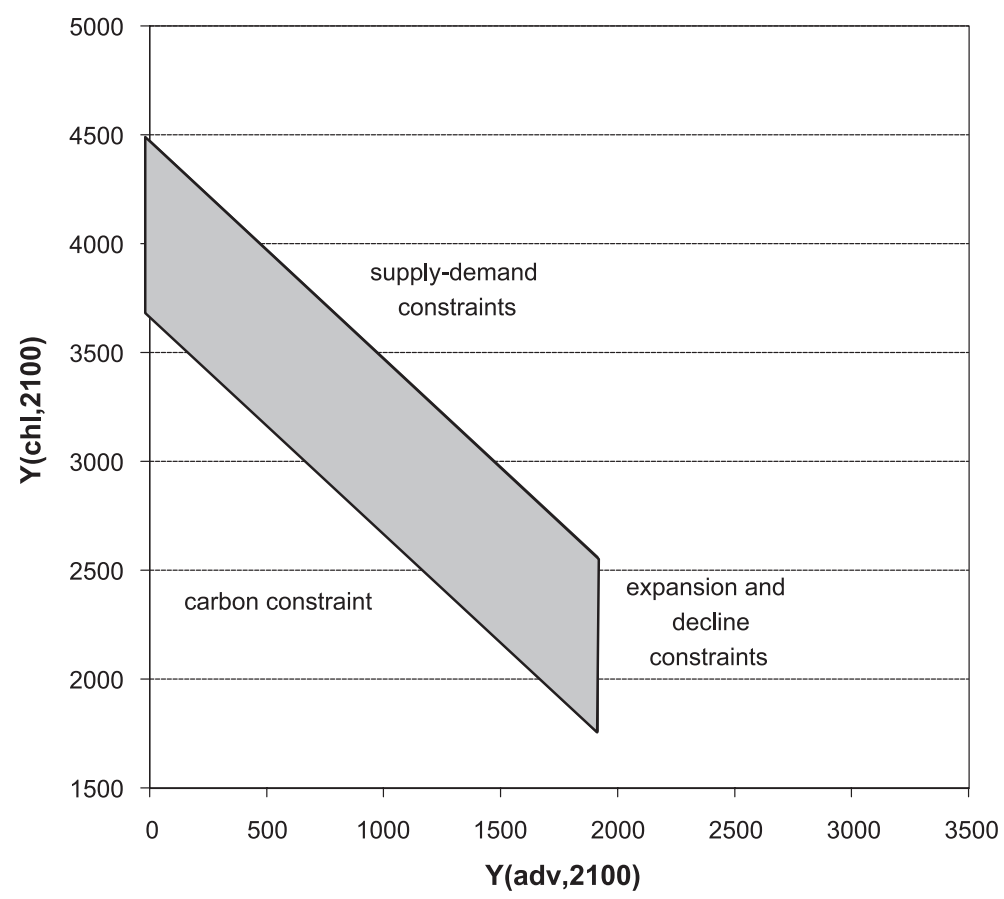

Fig. 6. Feasible combinations of the two cumulative experience variables.

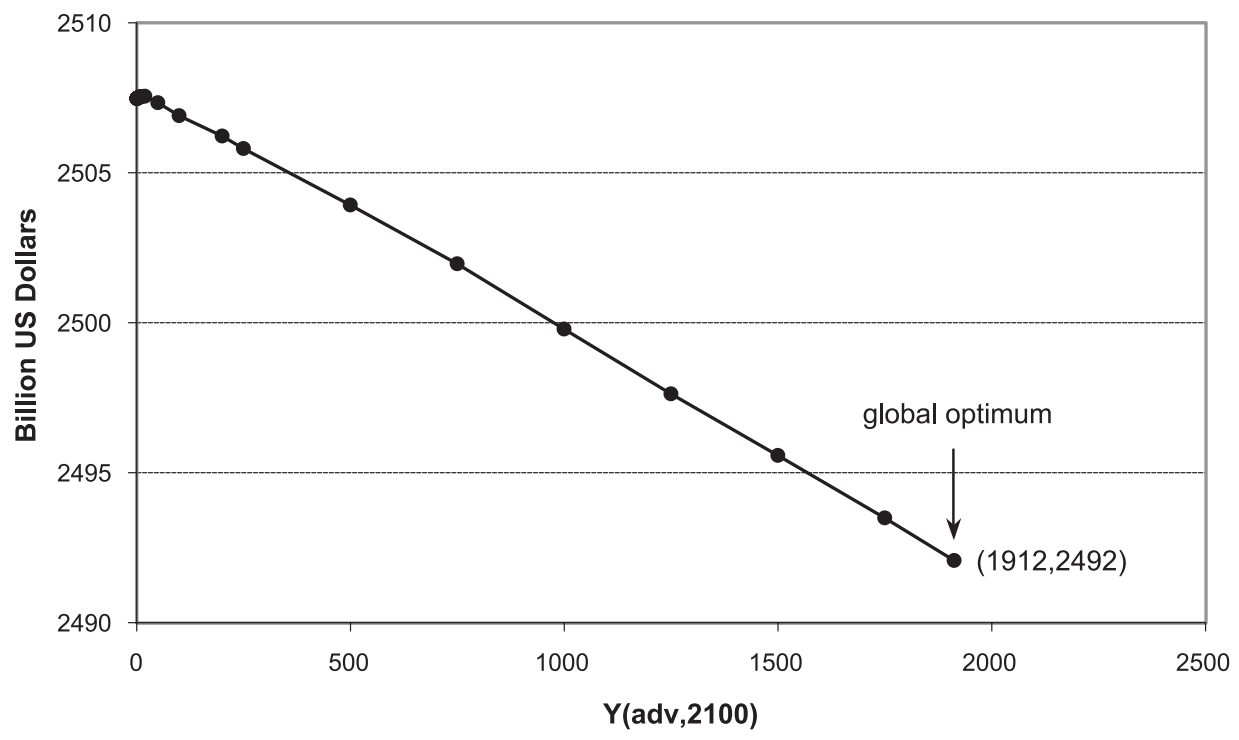

Fig. 7. Present value of costs (inlc(adv) increased to $\$ 20 / M W h)$. 


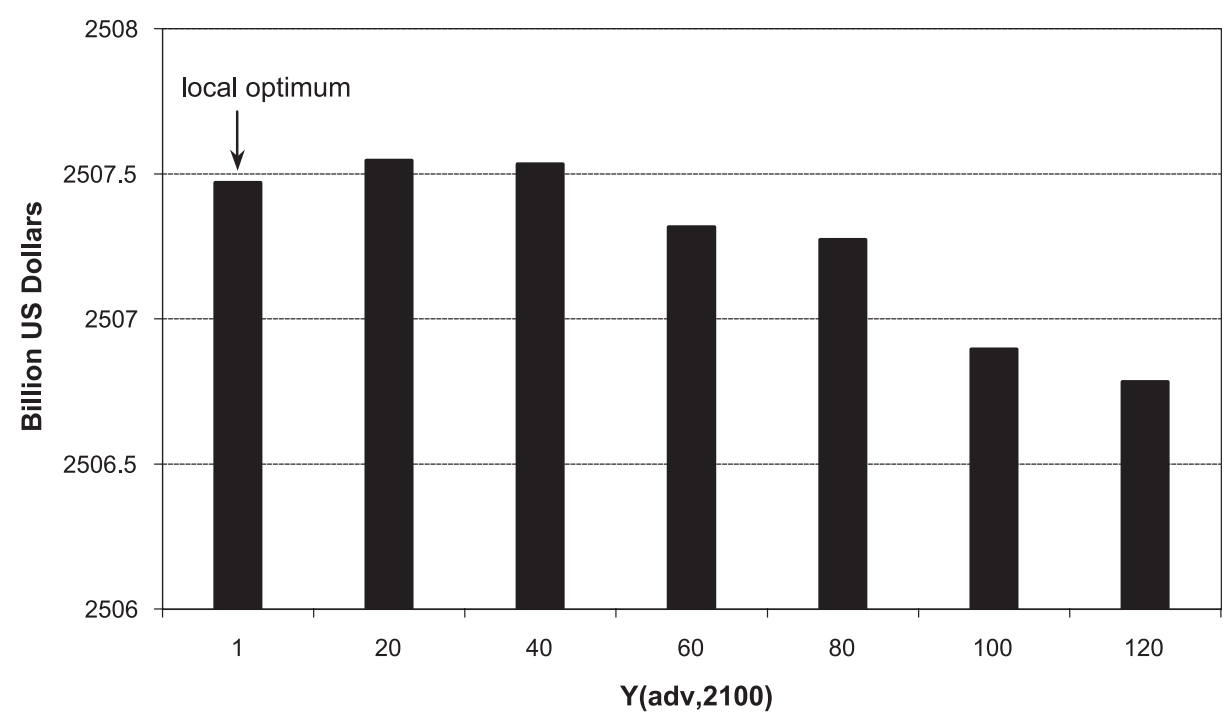

Fig. 8. Present value of costs - a closer look (inlc(adv) increased to $\$ 20 / \mathrm{MWh})$.

Now turn to Fig. 7. This shows how the minimum present value of costs varies when we alter the terminal value of the cumulative experience with the advanced technology. It looks as though there is no local minimum. This appears to be a monotone decreasing function, and the global minimum occurs when the advanced technology is brought in at a maximum level. However, when we take a closer look at the left-hand portion of this diagram (the stacked column graph shown in Fig. 8), there is a second local minimum. This occurs at the lowest admissible value of $Y(\operatorname{adv}, 2100)$. To avoid this local minimum, all that we need to do is to introduce an arbitrary lower bound on this decision variable. For example, with a lower bound of 20, we rule out the local solution at 1 , and the nonlinear solver CONOPT3 proceeds directly to the global optimum at the maximum value of 1912 (again see Fig. 7).

A similar procedure has been applied to MERGE, and it seems to work well. Caveat: In MERGE, there is only one LBD technology for the electric sector and one for the nonelectric sector. With several LBD technologies, these arbitrary bounds would have to be selected with greater care. There would then be considerable value in developing an algorithm such as BARON - one which is guaranteed to find a global optimum. Until such a procedure is developed, it will be useful to employ the terminal conditions heuristic.

\section{Acknowledgements}

Helpful comments have been received from Socrates Kypreos, Richard Richels, Michael Saunders, Leo Schrattenholzer, Arthur F. Veinott, Jr. and Clas-Otto Wene. For research assistance, the authors are indebted to Charles $\mathrm{Ng}$. 


\section{References}

Arrow, K., 1962. The economic implications of learning by doing. Review of Economic Studies. June.

Goulder, L., Mathai, K., 2000. Optimal $\mathrm{CO}_{2}$ abatement in the presence of induced technological change. Journal of Environmental Economics and Management. January.

Gritsevskyi, A., Nakicenovic, N., 2000. Modelling uncertainty of induced technological change. Energy Policy 28 , 907-921.

Hirsch, W.M., Hoffman, A.J., 1961. Extreme varieties, concave functions, and the fixed charge problem. Communications on Pure and Applied Mathematics XIV, 355-369.

International Energy Agency, 2000. Experience Curves for Energy Technology Policy. Organisation for Economic Co-operation and Development, Paris.

Kydes, A., 1999. Modeling technology learning in the national energy modeling systemIssues in Midterm Analysis and Forecasting 1999. Energy Information Administration, U.S. Department of Energy, Washington, USA.

Kypreos, S. (2000). "The MERGE Model with Endogenous Technological Change and the Climate Change", working paper, Paul Scherrer Institute. Presented at "Economic Modelling of Environmental Policy and Endogenous Technological Change Workshop", Amsterdam, November 16-17, 2000.

Kypreos, S., Barreto, L., Capros, P., Messner, S., 2000. ERIS: A model prototype with endogenous technological change. International Journal of Global Energy Issues 14. (1/2/3/4), 374-397.

Mattsson, N., Wene, C.-O., 1997. Assessing new energy technologies using an energy system model with endogenized experience curves. International Journal of Energy Research 21, 385-393.

Messner, S., 1997. Endogenised technological learning in an energy systems model. Journal of Evolutionary Economics 7, 291-313.

Sahinidis, N., 2000. BARON: Branch and Reduce Optimization Navigator. User's Manual Version 4.0. Department of Chemical Engineering. University of Illinois at Urbana-Champaign.

Seebregts, A., Bos, S., Kram, T., Schaeffer, G., 2000. Endogenous Learning and Technology Clustering: Analysis with MARKAL Model of the Western European Energy System. International Journal of Global Energy Issues $14(1 / 2 / 3 / 4), 289-319$.

TEEM (1999). "Energy Technology Dynamics and Advanced Energy System Modelling. Final Technical Report”. Contract JOS3 CT97 0013. Non-Nuclear Energy Programme JOULE III. European Commission.

Van der Zwaan, B.C.C., Gerlagh, R., Klaassen, G., Schrattenholzer, L., 2002. Endogenous technological change in climate change modelling. Energy Economics 24, 1-19. 



\section{Ordering Information}

Orders must include the publication number and should be sent to the Publications Department, International Institute for Applied Systems Analysis, A-2361 Laxenburg, Austria.

Telephone: +432236807

Telefax: +432236 71313

E-mail: publications@iiasa.ac.at

A full list of IIASA publications is available at www.iiasa.ac.at/Publications 
International Institute for Applied Systems Analysis

Schlossplatz 1, A-2361 Laxenburg, Austria

Tel: +432236 807 Fax: +43 223671313

I I AS A www.iiasa.ac.at 